\title{
Are renal microgranulomas related to inflammatory tubular destruction?
}

\author{
A Mahmood, D N Poller, J K Ramage
}

J Clin Pathol 2004;57:551-552. doi: 10.1136/jcp.2003.013722

Two cases of renal microgranuloma formation are reported, one in a patient with known Crohn's disease and another in a case of acute renal allograft rejection. In both cases, the microgranulomas arose as a result of inflammatory tubular destruction, in a manner analogous to that seen in patients with ulcerative colitis arising adjacent to ruptured epithelial crypts in the large intestine. Microgranulomas may occur at multiple anatomical sites in Crohn's disease, although renal microgranulomas are very rare. Non-specific inflammatory tubular destruction should be considered as a cause of renal microgranuloma formation, in addition to systemic granulomatous diseases, such as tuberculosis, sarcoid, or Crohn's disease, when granulomas are seen in the presence of inflammatory tubular destruction in renal biopsies.

W report two cases of renal microgranuloma formation, one in a patient with known Crohn's disease and the other in a case of acute renal allograft rejection. We suggest that renal microgranuloma formation may be caused by non-specific inflammatory tubular destruction.

\section{CASE 1}

A 41 year old woman with an initial diagnosis of ulcerative colitis and a 15 year history of symptoms developed recent onset non-bloody diarrhoea. The patient was taking sulfasalazine. Flexible sigmoidoscopy showed patchy colitis up to $30 \mathrm{~cm}$, with epithelioid microgranulomas and active inflammation, indicative of Crohn's disease. An ultrasound scan revealed slightly small kidneys with no evidence of obstruction or scarring. Because of impaired renal function, a renal biopsy was performed, which showed two sclerosed glomeruli and two normal glomeruli, with epithelioid granuloma formation and a florid tubulointerstitial nephritis, without evidence of extraglomerular vasculitis. The granulomas appeared to arise as a result of inflammatory tubular destruction (fig 1). Chest radiograph, serum angiotensin convertase values, vasculitis screen, myeloma profile, and immunoglobulin values, including IgA, were all normal. No tubercle bacilli were present in the urine. She was started on high dose prednisolone, but this had no impact on the renal function.

\section{CASE 2}

A 74 year old man had chronic renal failure of unknown aetiology. At the age of 30, it had been discovered that his right kidney and ureter were absent. There was no history of granulomatous systemic disease. He had been on peritoneal dialysis for approximately one year before the renal transplant. Two weeks after transplantation his renal function deteriorated. A renal biopsy demonstrated acute cellular rejection (fig 2), which was suppressed with pulsed methyl prednisolone. Post transplant immunosuppression comprised cyclosporine, azathioprine, and prednisolone.

\section{DISCUSSION}

In both the above cases the renal microgranulomas were seen adjacent to tubules showing evidence of inflammatory tubular destruction, in one case as a result of tubulointerstitial nephritis, possibly related to sulfasalazine treatment, and in the second case as a result of renal allograft rejection. Granulomas arising as a result of inflammatory destruction of large intestinal crypts occur in mucosal biopsies of the large intestine in ulcerative colitis. ${ }^{12}$ The absolute sensitivity and specificity of the demonstration of granulomas in the diagnosis of intestinal Crohn's disease has been questioned because granulomas can occur as a result of a tissue response to mucin, presumably arising as a result of crypt obstruction, inflammation, and disruption. Archimandritis and Weetch reported renal involvement in Crohn's disease several years ago, demonstrating interstitial nephritis with granuloma formation. ${ }^{3}$ However, in our opinion, granulomas in the kidney involving destroyed renal tubules are not always unequivocal evidence of a systemic granulomatous disease, such as extraintestinal Crohn's disease. In any case, extraintestinal renal involvement in Crohn's disease with renal granuloma formation is rare. ${ }^{3}$

\section{"Granulomas arising as a result of inflammatory destruc- tion of large intestinal crypts occur in mucosal biopsies of the large intestine in ulcerative colitis"}

Sulfasalazine may have caused the interstitial nephritis in case 1, because it is associated with interstitial nephritis, particularly after prolonged exposure, although it has not previously been associated with reports of tubule related renal granuloma formation. Sulfasalazine is a 5 -aminosalicylate (5-ASA) linked by a diazo bond to the carrier sulfapyridine, which acts primarily via 5-ASA released by bacterial cleavage of the diazo bond in the colon. Dwarakanath et al reported sulfasalazine induced renal failure in two patients. ${ }^{4}$ Both patients had taken sulfasalazine for several years. The renal biopsies were consistent with drug induced interstitial nephritis and no granulomata were seen in either case. One patient required renal transplantation, the other had impaired but stable renal function. Calvino et al reported mesalazine related tubulointerstitial nephritis, which failed to improve despite withdrawal of mesalazine and a trial of steroids. ${ }^{5}$

Interstitial nephritis may also be a non-specific manifestation of Crohn's disease. Izzedine et al reported four patients with Crohn's disease and interstitial nephritis; ${ }^{6}$ in all four cases there was granuloma formation, although the granulomas were not described in association with ruptured or

Abbreviations: 5-ASA, 5-aminosalicylate 

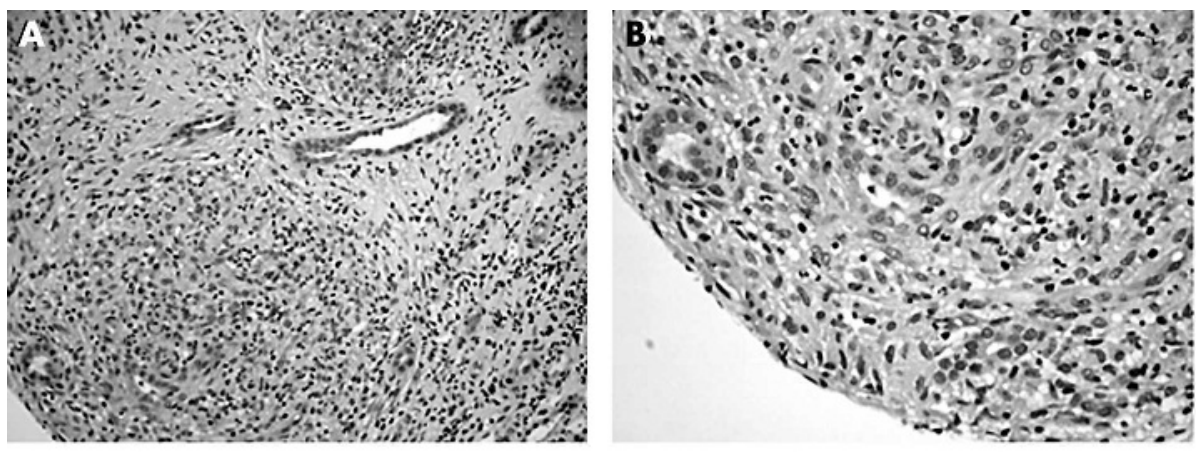

Figure 1 Case 1. (A) Low power photomicrograph of renal medulla showing a poorly formed granuloma with several disrupted and destroyed renal tubules within the centre of the granuloma. (B, C) Higher power photomicrographs showing the histiocytes forming a granuloma intimately associated with the disrupted and destroyed renal tubules. (D) Low power photomicrograph showing renal cortical tubules elsewhere within the same biopsy, which appeared unremarkable. A mild interstitial
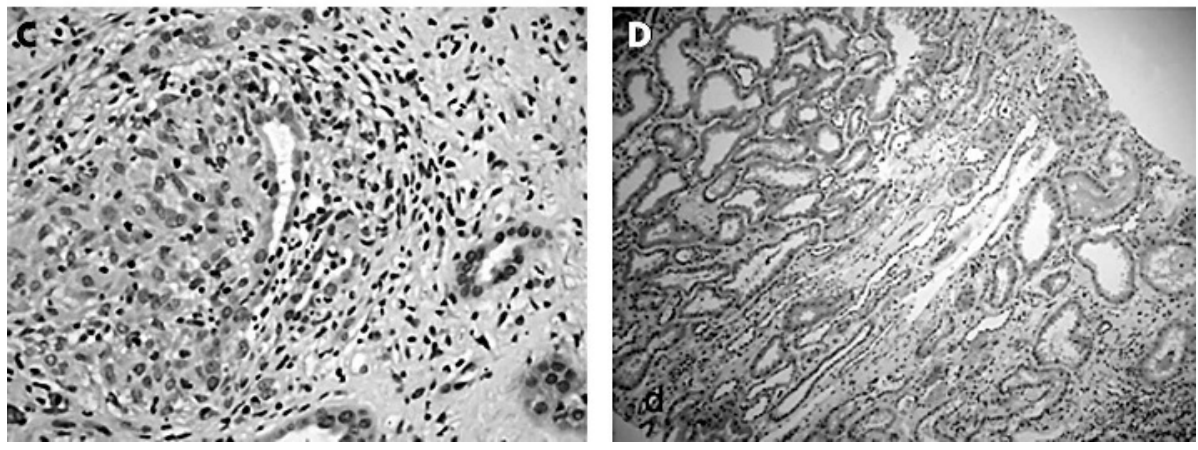
lymphocytic nephritis is present.

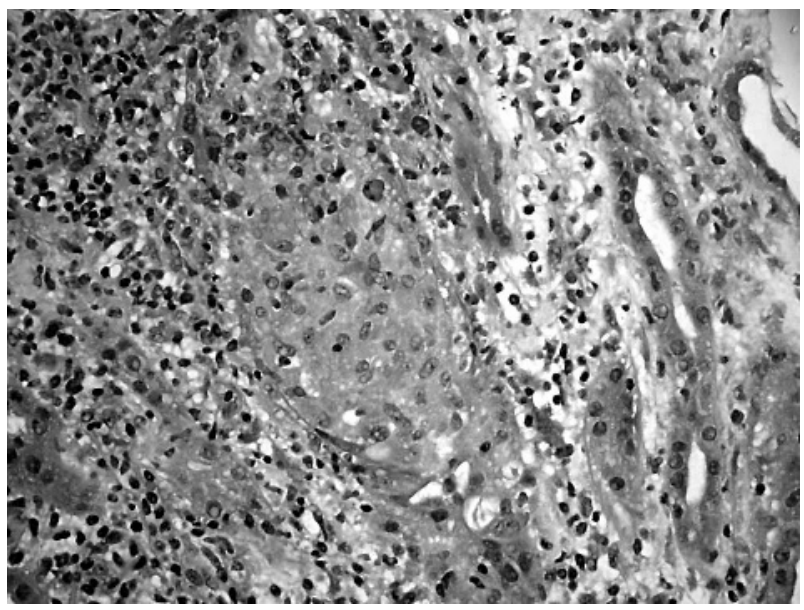

Figure 2 Case 2. Renal tubule showing a granulomatous response arising presumably as a result of T cell tubular destruction in a patient with acute cellular allograft rejection.

destroyed renal tubules and no 5-ASA compounds were given. In case 2, the granulomatous inflammation was

\section{Take home messages}

- We report two cases of renal microgranuloma formation arising as a result of inflammatory tubular destruction, one in a patient with Crohn's disease who was taking sulfasalazine and the other in acute renal allograft rejection

- Non-specific inflammatory tubular destruction should be considered as a cause of renal microgranuloma formation, in addition to systemic granulomatous diseases (such as tuberculosis, sarcoid, or Crohn's disease), when granulomas are seen in the presence of inflammatory tubular destruction in renal biopsies presumed to be caused by $\mathrm{T}$ cell induced lymphocytic destruction of tubules in a patient with acute cellular renal allograft rejection. The inflammatory tubular destruction evident in the two renal biopsies presumably gave rise to the granulomas seen in both cases that were seen in relation to destroyed renal tubules, in a manner analogous to that causing granuloma formation in the large intestine. ${ }^{12}$

In summary, when granulomas are identified in the kidney, arising as a result of inflammation adjacent to destroyed renal tubules, inflammatory renal tubular destruction should be considered as a cause of granuloma formation, in addition to other potential causes, such as systemic granulomatous disease.

\section{ACKNOWLEDGEMENTS}

Thanks to Dr J Stevens for permission to report case 1 and Mr SA Sadek for permission to report case 2 .

\section{Authors' affiliations}

A Mahmood, Department of Gastroenterology, Queen Alexandra Hospital, Portsmouth, PO6 3LY, UK

D N Poller, Department of Pathology, Queen Alexandra Hospital J K Ramage, Department of Gastroenterology, North Hampshire Hospital, Basingstoke, RG24 9NA, UK

Correspondence to: Dr D N Poller, Department of Pathology, Queen Alexandra Hospital, Cosham, Portsmouth, PO6 3LY, UK; david.poller@ porthosp.nhs.uk

Accepted for publication 27 October 2003

\section{REFERENCES}

1 Shepherd NA. Granulomas in the diagnosis of intestinal Crohn's disease: a myth exploded? Histopathology 2002;41:166-8.

2 Shepherd NA. Pathological mimics of chronic inflammatory bowel disease. J Clin Pathol 1991;44:726-33.

3 Archimandritis AJ, Weetch MS. Kidney granuloma in Crohn's disease. BMJ 1993;307:540-1.

4 Dwarakanath AD, Michael J, Allan RN. Sulphasalazine induced renal failure. Gut 1992;33:1006-7.

5 Calvino J, Romero R, Pintos E, et al. Mesalazine associated tubulo-interstitial nephritis in inflammatory bowel disease. Clin Nephrol 1998;49:265-7.

6 Izzedine H, Simon J, Piette A, et al. Primary chronic interstitial nephritis in Crohn's disease. Gastroenterology 2002;123:1436-40. 